\title{
Safety and Efficiency of Percutaneous Dilatational Tracheostomy With Direct Bronchoscopic Guidance for Thoracic Transplant Recipients
}

\author{
Kevin Pilarczyk MD, Henning Carstens MD, Jens Heckmann MD, Juri Lubarski MD, \\ Günter Marggraf MD, Heinz Jakob MD PhD, Nikolaus Pizanis MD, and Markus Kamler MD PhD
}

\begin{abstract}
BACKGROUND: Percutaneous dilatational tracheostomy (PDT) is the standard airway access in critically ill patients who require prolonged mechanical ventilation. However, the literature lacks reports about the effectiveness and safety of this procedure in thoracic organ transplant recipients, who have increased risks of bleeding and infection. METHODS: We retrospectively reviewed the records of subjects who underwent thoracic organ transplantation at our institution between January 2004 and March 2011 followed by PDT (using the Ciaglia Blue Rhino technique with direct bronchoscopic guidance). RESULTS: From a total of $\mathbf{3 1 2}$ thoracic transplant recipients, we identified $93(29.8 \%)$ subjects with PDT. Of these, 79 had undergone double lung transplant, 11 had undergone heart transplant, 2 had undergone combined heart-lung transplant, and 1 had undergone combined heart-kidney transplant. Mean age was $49.5 \pm 11.2 \mathrm{y}$, and $58 \%$ of subjects were female. The mean time from intubation to PDT was $3.7 \pm 3.4 \mathrm{~d}$, and mean time from transplant to PDT was 12.6 $\pm 28.3 \mathrm{~d}$. Thirty-two subjects $(34.4 \%)$ underwent PDT after re-intubation. Thirtynine subjects were receiving renal replacement therapy $(41.9 \%)$, and 28 had a coagulopathy $(30.1 \%)$. Moderate but not significant bleeding was observed in 3 subjects. There were no major complications during PDT procedures. Forty-five subjects $(48.4 \%)$ could be weaned successfully from the ventilator and the tracheostoma could be removed. Forty-eight subjects $(51.6 \%)$ died due to sepsis, multi-organ failure, or transplant failure. No procedure-related deaths were noted. There were no significant late complications. Among the 45 who survived their stay in the ICU, the functional and cosmetic outcomes of PDT were excellent. CONCLUSIONS: PDT can be safely performed on patients with acute respiratory failure after thoracic organ transplantation. Therefore, we recommend the use of this technique for prolonged airway management in these patients. Key words: percutaneous dilatational tracheostomy; thoracic transplantation; lung transplantation; cardiac transplantation; airway management. [Respir Care 2016;61(2):235-242. ( 2016 Daedalus Enterprises]
\end{abstract}

\section{Introduction}

Tracheostomy is regularly considered for airway access in critically ill patients requiring prolonged mechanical

\footnotetext{
Dr Pilarczyk is affiliated with the Department of Intensive Care Medicine, Imland Klinik Rendsburg, Rendsburg, Germany. Drs Carstens, Heckmann, Lubarski, Marggraf, Jakob, Pizanis, and Kamler are affiliated with the Department of Thoracic and Cardiovascular Surgery, West German Heart and Vascular Center Essen, University Hospital Essen, Essen, Germany.
}

The authors have disclosed no conflicts of interest. ventilation to facilitate adequate airway management and ventilatory weaning. Since the introduction of guidewireassisted percutaneous dilational tracheostomy (PDT) into routine clinical practice by Ciaglia et al, ${ }^{1}$ this technique or its modifications have been used increasingly in different patient populations. ${ }^{2}$ This technique has several advan-

Correspondence: Kevin Pilarczyk MD, Department of Intensive Care Medicine, Imland Klinik Rendsburg, Rendsburg, Germany. E-mail: kevin.pilarczyk@uk.essen.de.

DOI: $10.4187 /$ respcare. 04128 
tages over the traditional surgical tracheostomy. It is easier to perform, less expensive, and shorter, with less need for sedation and analgesia. ${ }^{2}$ Moreover, it can be performed in the ICU, so the unstable patient does not have to be transferred to the operative theater. In addition, some authors have suggested a lower rate of complications like infections or bleeding. ${ }^{3}$

Patients after transplantation have an increased risk of systemic infections, pneumonia, and ARDS, which in turn can lead to respiratory failure requiring prolonged mechanical ventilation. This applies in particular to lung transplantation, which has a higher incidence of infections and acute rejection episodes than any other form of solid organ transplantation. ${ }^{4}$ It is generally presumed that tracheostomy can increase the risk for mediastinitis in patients undergoing cardiac surgery with median sternotomy with a possible cross-contamination of the tracheostomy and sternotomy wounds due to its close proximity. Heart transplant recipients undergoing PDT might be more susceptible to deep sternal infections due to their immunosuppressive therapy. Furthermore, coagulopathy is frequently observed in transplant patients, considered as contraindication to tracheostomy by many authors.

Limited data are available in the transplant literature about the safety of PDT for lung and heart allograft recipients, who have increased risk of complications such as bleeding or infection due to immunosuppressive therapy. ${ }^{5,6}$ Therefore, it was the aim of our study to assess the safety and efficacy of PDT in lung and heart transplant recipients experiencing prolonged ventilation.

\section{Methods}

\section{Subjects}

Between January 1, 2004, and March 31, 2011, data were prospectively collected on all consecutive lung and heart transplantations at our university hospital, Department of Thoracic and Cardiovascular Surgery, West German Heart and Vascular Center Essen. A retrospective analysis was then performed. The present study obtained institutional review board approval according to the Declaration of Helsinki.

\section{Transplantation Procedure}

Lung procurement and preservation followed standard procedures with cold Perfadex solution for flushing. The technical details for the lung transplant procedure have been described in detail elsewhere. ${ }^{7}$ All bilateral lung transplants were performed with standard use of cardiopulmonary bypass. Immunosuppressive induction therapy was performed by administration of ATG (Thymoglobuline, Imtix Sangstat, Lyon, France) at a dose of $2.5 \mathrm{mg} / \mathrm{kg}$ for a

\section{QUICK LOOK}

\section{Current knowledge}

Tracheostomy is commonly used in critically ill patients requiring prolonged mechanical ventilation to facilitate adequate airway management and discontinuation of ventilator support. Percutaneous dilational tracheostomy (PDT) in the ICU has become a common method of tracheostomy placement. PDT has been shown to be easier to perform and less expensive, requires less sedation and analgesia, and can be accomplished without transferring the patient to the operating room.

\section{What this paper contributes to our knowledge}

PDT was used as the standard airway access for subjects requiring prolonged mechanical ventilation after lung or heart transplant. The procedure was safe and had a low complication rate. Bleeding or infectious complications due to immunosuppressive therapy and coagulopathy were rare.

period of $3 \mathrm{~d}$. Immunosupression was based on triple therapy (cyclosporine, mycophenolate mofetil, and prednisolone). Prophylaxis for Pneumocystis carinii and cytomegalievirus infection was achieved with low-dose oral thrimethroprim-sulfamethoxazole and intravenous ganciclovir, respectively. Standardized evaluation for rejection and infection included clinical assessment, chest radiograph, and pulmonary function tests. Diagnostic bronchoscopy was performed daily in the immediate postoperative period and was performed subsequently for clinical symptoms or spirometric evidence of allograft dysfunction. Surveillance bronchoscopy and transbronchial biopsies were performed after $3,6,9,12,18$, and 24 months in the absence of symptoms.

The most recent laboratory data (from tests conducted within $12 \mathrm{~h}$ before PDT) were collected and included prothrombin time, activated partial thromboplastin time, platelet count, and hemoglobin level. Abnormal coagulation profiles were defined as: (1) platelets $<50,000 / \mu \mathrm{L}$ (2) prothrombin time $>50 \%$ of the normal reference value, (3) activated partial thromboplastin time $>50 \mathrm{~s}$. The administration of intravenous unfractionated heparin was stopped $2 \mathrm{~h}$ before the planned tracheostomy without further control of activated partial thromboplastin time before the procedure. All blood samples were drawn before stopping heparin administration. Heparin was recommenced immediately after completion of the procedure at the previous rate and adjusted to subsequent coagulation test results. PDT was performed without stopping antiplatelet agents if 
there was a clear and ongoing indication for the antiplatelet agent.

\section{Performance of PDT}

No surgical tracheostomies were performed during the study period, and no subjects were excluded. All procedures were performed at the bedside in the intensive care unit by a cardiothoracic surgeon or intensivist. Two physicians and at least one nurse were always present; the cardiothoracic surgery team provided surgical cover. PDT was performed using the Ciaglia Blue Rhino technique (Tracoe Medical, Nieder-Olm, Germany) with direct bronchoscopic guidance. One physician provided video bronchoscopic guidance, and the other performed the procedure. Tracheostomies were performed by an experienced consultant (at least 50 PDTs performed) or a senior ICU trainee under consultant supervision. Informed consent from the subject's next of kin was obtained to undertake the percutaneous tracheostomy. The indication for tracheostomy was made by the consultant in charge of the ICU at the time. The indication for tracheostomy was respiratory failure attributed to a variety of underlying medical conditions, most commonly exacerbation of chronic lung disease, muscle weakness, neurological deterioration, pulmonary infection, and acute lung injury with an expected length of mechanical ventilation exceeding 7-10 d. Prediction of need for prolonged mechanical ventilation was mainly based on the clinical experience and subjective physician evaluation. Subjects were sedated and analgesed as needed. Heparin was discontinued approximately $2-3 \mathrm{~h}$ before PDT without additional laboratory control of the coagulation status. Anti-platelet therapy was not paused. Commonly used sedatives were remifentanil, propofol, and midazolam. Muscle relaxation was used in all subjects, and neuromuscular blockade was achieved in most cases by using rocuronium. $\mathrm{F}_{\mathrm{IO}_{2}}$ was increased to 1.0 , and ventilator settings were kept unchanged, apart from subjects receiving pressure support ventilation, in which a controlled mode of ventilation was initiated for the period of the intervention and maintained until reversal of paralysis. Continuous monitoring of heart rate, arterial oxygen saturation, and invasive arterial blood pressure was performed in all subjects. Resuscitation and difficult airway equipment was present at the bedside.

Subjects were placed in the supine position. Their necks were extended with folded sheets and were prepared and draped in a sterile manner. Flexible bronchoscopic visualization was routinely utilized to facilitate insertion in all subjects. A bronchoscope was advanced to the end of the endotracheal tube. After deflating the cuff, the endotracheal tube was slowly withdrawn in concert until the bronchoscope light illuminated the planned tracheostomy site or digital manipulation of the trachea over the site could be seen through the bronchoscope.

A 16-gauge introducer needle was advanced into the trachea in the midline between the first and second or the second and third tracheal cartilage rings. A J-tipped wire was advanced inferiorly through the needle, and the introducer needle was removed. A small skin incision (approximately $1.0-1.5 \mathrm{~cm}$ ) was made with a scalpel at the entry site of the wire. A 14 French introducer dilator was then advanced. One large hydrophilic dilator was advanced over the guiding catheter and wire until the stoma was appropriately dilated for passage of the cannula and the dilatator was removed. A tracheostomy cannula loaded onto a guiding catheter was advanced over the J-wire into the trachea. The guiding catheter and J-tipped wire were removed, leaving the tracheostomy cannula in place. After balloon insufflation, ventilation was switched from the endotracheal tube to the tracheostomy tube. Regions of the trachea superior and inferior to the cannula were examined, blood was aspirated if needed, and the endotracheal tube was removed. Typically, the tracheostomy tube was secured with neck ties; sutures were not used. After completion of the PDT, a chest radiograph was performed to rule out a pneumothorax and other abnormalities. Heparin was initiated $2 \mathrm{~h}$ after completion of the procedure at the previous rate and adjusted to subsequent coagulation test results.

All data acquired during the subjects' surveillance were transferred to our institutional database. Coagulopathy/thrombocytopenia was defined as one or more of the following: (1) platelets $<50,000 / \mu \mathrm{L}$ (2) prothrombin time $>50 \%$ of the normal reference value, and/or (3) activated partial thromboplastin time $>50 \mathrm{~s}$.

\section{Assessment of Complications}

Complications related to PDT, such as death, hypoxemia, pneumothorax, bleeding, tracheal injuries (posterior wall perforation, fractures of tracheal rings), cannula dislocation, premature decannulation, and stomal infections were documented. The extent of intrabronchial bleeding was graded as mild (1-2 blood-covered sponges or the presence of some blood on the posterior tracheal wall not requiring intratracheal suction), moderate (PDT site bloodcovered or segmental bronchus obstructed by blood), severe (main stem bronchus obstructed by blood), or major (surgical intervention/transfusion required). Extratracheal bleeding was classified as mild $(1-5 \mathrm{~mL})$, moderate $(5-10 \mathrm{~mL})$, severe $(11-50 \mathrm{~mL})$, or major $(>50 \mathrm{~mL}$ and/or surgical intervention/transfusion required). Insertion of the tracheostomy tube was graded as easy, moderately difficult, very difficult, or impossible. Grading of PDT difficulty was based on subjective assessment of the performing physician rather than objective criteria. Mean 


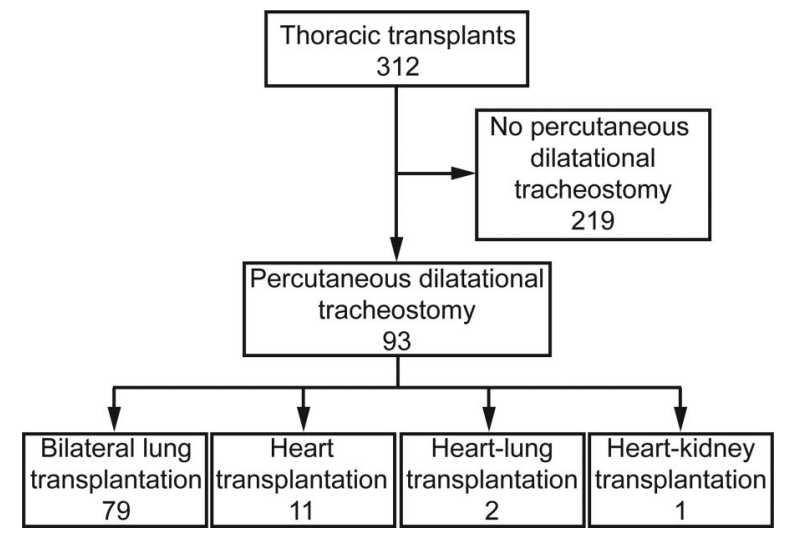

Fig. 1. Flow chart.

follow-up was $1,014 \pm 838 \mathrm{~d}$ (median 751 [range $0-3,376])$.

\section{Results}

\section{Subject Demographics}

Between January 1, 2004, and March 31, 2011, we identified 312 thoracic transplantations at our institution. During the study period, a total of 93 subjects (29.8\%) were scheduled for tracheostomy in our cardiothoracic ICU. No subjects were assigned for primary open surgical tracheostomy. Therefore, 93 subjects (100\%) underwent PDT with a success rate of $100 \% ; 79$ subjects $(84.9 \%)$ had undergone double lung transplant, 11 (11.8\%) had undergone heart transplant, $2(2.2 \%)$ had undergone combined heart-lung transplant, and $1(1.1 \%)$ had undergone combined heart-kidney transplant (see Fig. 1).

Table 1 shows the clinical profile of tracheostomy subjects. Mean age was $49.5 \pm 11.2$ y (range $18-67 \mathrm{y}$ ), and $58.2 \%$ of subjects were female $(n=46)$. Twenty-three subjects were receiving renal replacement therapy when tracheostomy was performed. Terminal laboratory findings, including the coagulation status before performance of PDT, are summarized in Table 2.

PDT was performed in 28 subjects (30\%) with a coagulopathy. Activated clotting time, platelets, partial thromboplastin time, and international normalized ratio were $124.7 \pm 24.2 \mathrm{~s}, 119.8 \pm 115.8 \times 1,000 / \mu \mathrm{L}, 43.4 \pm 18.8 \mathrm{~s}$, and $1.7 \pm 0.8 \mathrm{~g} / \mathrm{dL}$ (Table 2). All blood samples were drawn before stopping heparin administration $2 \mathrm{~h}$ before PDT.

Table 1 provides a detailed description of primary diagnoses and indices of severity of illness. The most common reason for lung transplantation was emphysema ( $n=43,54.4 \%$ ), followed by pulmonary fibrosis $(n=20$, $25.3 \%$ ). Severity of illness in the lung transplant group is indicated by mean duration of waiting time $(516 \pm 417 \mathrm{~d})$,
Table 1. Preoperative Data of Subjects

\begin{tabular}{lc}
\hline \hline \multicolumn{1}{c}{ Preoperative Characteristics } & Values \\
\hline Age, mean \pm SD y & $49.5 \pm 11.2$ \\
Female sex, $n(\%)$ & $46(58.2)$ \\
BMI, mean \pm SD kg/m ${ }^{2}$ & $23.2 \pm 5.3$ \\
Indication for lung transplant, $n(\%)$ & \\
Emphysema & $43(54.4)$ \\
Idiopathic pulmonary fibrosis & $20(25.3)$ \\
Cystic fibrosis & $9(11.4)$ \\
Primary pulmonary hypertension & $2(2.5)$ \\
Redo transplant for chronic graft failure & $3(3.8)$ \\
Others & $2(2.5)$ \\
High-urgency status on waiting list, $n(\%)$ & $50(63.3)$ \\
Time on waiting list, mean \pm SD d & $516 \pm 417$ \\
Time on high-urgency status, mean \pm SD d & $37 \pm 45$ \\
Preoperative mechanical ventilation, $n(\%)$ & $18(22.7)$ \\
\end{tabular}

BMI = body mass index

Table 2. Coagulative Status

\begin{tabular}{lc}
\hline \hline \multicolumn{1}{c}{ Coagulation Test } & Mean $\pm \mathrm{SD}$ \\
\hline Activated clotting time, s & $124.7 \pm 24.2$ \\
Platelets, $\times 1,000 / \mu \mathrm{L}$ & $119.8 \pm 115.8$ \\
Partial prothrombin time, s & $43.4 \pm 18.8$ \\
Prothrombin time, $\%$ normal reference & $81.3 \pm 22.5$ \\
INR & $1.7 \pm 0.8$ \\
Fibrinogen, g/dL & $425.4 \pm 193.8$ \\
Coagulopathy, $n(\%) *$ & $28(30)$ \\
& \\
$*$ Defined as one or more of the following criteria met: (1) platelets $<50.000 / \mu \mathrm{L},(2)$ \\
prothrombin time $>50 \%$ of the normal reference value, (3) activated partial thromboplastin \\
time $>50$ s. \\
INR $=$ international normalized ratio \\
\hline
\end{tabular}

incidence of high urgency status ( $n=50,63.3 \%$ ), or preoperative mechanical ventilation $(n=18,22.8 \%)$.

\section{Performance of PDT}

The indication for tracheostomy was respiratory failure attributed to a variety of underlying medical conditions, most commonly muscle weakness, neurological deterioration, pulmonary infection, and acute lung injury. The majority of PDT tube insertion was graded as easy $(n=77$, $83 \%$ ). Only 3 cases were classified as very difficult (Table 3 ). Tracheostomy was completed successfully in all subjects, and no subject had to be converted to open tracheostomy. The majority of all tubes were placed between the second and third or between the third and fourth ring, respectively ( $n=69,74.2 \%$ ). PDT was performed a mean of $12.6 \pm 28.3 \mathrm{~d}$ after transplantation. In 3 subjects, PDT was performed after initial discharge from the hospital and 
Table 3. Overview of Techniques Required During Percutaneous Dilatational Tracheostomy

\begin{tabular}{lc}
\hline \hline \multicolumn{1}{c}{ Technique } & $n(\%)$ \\
\hline Puncture site between & \\
1st/2nd ring & $17(18)$ \\
2nd/3rd ring & $47(50)$ \\
3rd/4th ring & $22(24)$ \\
Others & $7(8)$ \\
Difficulty of insertion & \\
Easy & $77(83)$ \\
Moderately difficult & $13(14)$ \\
Very difficult & $3(3)$ \\
Impossible & $0(0)$ \\
Tracheostomy tube used & \\
8-mm ID & $45(48)$ \\
9-mm ID & $48(52)$ \\
Success rate & $93(100)$ \\
Conversion to open tracheotomy & $0(0)$ \\
\hline ID $=$ inner diameter & \\
\hline
\end{tabular}

readmission after 134,183 , and $542 \mathrm{~d}$. In all other subjects, PDT was done in the early postoperative course. Mean duration of mechanical ventilation before PDT was $3.75 \pm 3.40 \mathrm{~d}$ with a range from 0 to $16 \mathrm{~d}$. In $34.4 \%$ of all cases ( 32 subjects), percutaneous tracheostomy was performed after re-intubation for postextubation respiratory failure.

There were no deaths associated with PDT, and no subjects suffered from major complications, including cardiac arrest or pneumothorax (Table 4). No or only mild endotracheal bleeding was observed in $48 \%(n=45)$ and $47 \%$ $(n=44)$. The incidence of moderate bleeding was low, at $3 \%(n=3)$. Severe endotracheal bleeding was recorded in 1 subject in the early postinterventional course. However, bleeding stopped immediately after insertion of the tracheostomy tube without further treatment; no blood transfusions had to be given.

In the majority of subjects, no extratracheal bleeding was observed $(n=67,72 \%)$. Mild or moderate extracheal bleeding occurred in $24(26 \%)$ and $2(2 \%)$ subjects. No major bleeding complications requiring transfusion or surgery were observed.

We found no association between bleeding complications and platelet count, international normalized ratio, or partial thromboplastin time. Fractures of the tracheal ring could be observed in $20 \%$ of the subjects $(n=19)$ without any effect on further clinical course. Late complications were observed infrequently and included 1 granuloma, 1 purulent stomal infection, and 2 cases of dislocation of the tube. Fortyfive subjects $(48.4 \%)$ could be weaned successfully from the ventilator and the tracheostoma could be removed. Fortyeight subjects (51.6\%) died due to sepsis, multi-organ failure,
Table 4. Complications After Percutaneous Dilatational Tracheostomy

\begin{tabular}{lc}
\hline \hline \multicolumn{1}{c}{ Complications } & Incidence, $n(\%)$ \\
\hline Early complications & \\
Death & $0(0)$ \\
Cardiac arrest & $0(0)$ \\
Pneumothorax & $0(0)$ \\
Hypotension & $0(0)$ \\
Hypoxemia & $0(0)$ \\
Endotracheal bleeding & \\
None & $45(48)$ \\
Mild & $44(47)$ \\
Moderate & $3(3)$ \\
Severe & $1(1)$ \\
Extratracheal bleeding & \\
None & $67(72)$ \\
Mild & $24(26)$ \\
Moderate & $2(2)$ \\
Severe & $0(0)$ \\
Major bleeding (requiring surgery or transfusion) & $0(0)$ \\
Accidental extubation & $0(0)$ \\
Dislocation/accidental decannulation & $0(0)$ \\
Misplacement & $0(0)$ \\
Posterior wall lesion & $0(0)$ \\
Fracture of tracheal rings & $19(20)$ \\
Stomal infection (purulent) & \\
Mediastinitis & $1(1)$ \\
Tracheal stenosis & $0(0)$ \\
Granuloma & $0(0)$ \\
Tracheoesophageal fistula & $1(1)$ \\
& $0(0)$ \\
\hline
\end{tabular}

Table 5. Outcome After Percutaneous Dilatational Tracheostomy

\begin{tabular}{lc}
\hline \hline Outcome & Incidence \\
\hline Decannulated, $n(\%)$ & $45(48.4)$ \\
Before discharge & $44(47)$ \\
After discharge & $1(1)$ \\
ICU stay, mean \pm SD d & \\
Overall & $48.5 \pm 41.8$ \\
After tracheostomy & $35.9 \pm 32.9$ \\
Survival, $n(\%)$ & \\
ICU & $45(48.4)$ \\
$1 \mathrm{y}$ & $39(41.9)$ \\
\hline
\end{tabular}

or transplant failure. One year-survival was comparable with hospital mortality with $41.9 \%$. (Table 5)

\section{Discussion}

In the present study, the complication rate of PDT in thoracic transplant recipients with a potential risk of infections and bleeding was low and comparable with that of other patient populations in the literature, even when per- 
formed by residents under supervision by experienced consultants. Therefore, we recommend using this airway access as the first choice in patients after thoracic transplant with prolonged mechanical ventilation.

Several advantages have been suggested for tracheostomy in comparison with translaryngeal intubation, including superior weaning from mechanical ventilation in patients receiving long-term ventilatory support, facilitated nursing care, decreased risk of cannula displacement, and improved patient comfort. ${ }^{2}$ Older observational studies and single-center randomized controlled trials showed that tracheostomy not only decreases the need for sedation and facilitates weaning but also decreases subjects' time in the intensive care unit as well as mortality and improves mobilization. ${ }^{8}$ However, more recent randomized studies, including the TracMan study ${ }^{10}$ (the first multi-center study) could not detect any effect of timing of PDT on mortality, and recent meta-analyses suggest that evidence is of low quality and that further randomized, controlled trials are necessary to investigate differences between early and late PDT. 9-11 $^{-11}$

The technique of PDT offers numerous theoretical advantages over the surgical approach. PDT can be performed at bedside in the ICU, eliminating the risk of transferring critically ill patients to the operating room. In addition, limited dissection may translate to less tissue damage and a lower incidence of wound infection. PDT also results in important cost savings compared with surgical tracheostomy, by avoiding human resources and equipment for operating theater time. Because the operation schedule does not have to be considered for PDT, the time interval between decision-making and performing tracheotomy may be reduced.

Large randomized trials as well as meta-analysis could not persistently demonstrate any difference in complications rates between PDT and surgical tracheotomy. A metaanalysis of 17 randomized controlled studies including 1,212 subjects from 2006 found a significantly reduced wound infection rate of $2.3 \%$ after bedside PDT versus $10.7 \%$ following surgical tracheostomy either bedside or in the operating room. ${ }^{12}$ In addition, a subgroup analysis in which both procedures were performed in the operating room showed a reduction in bleeding and mortality with PDT. A meta-analysis by Higgins and Punthakee ${ }^{13}$ of 15 prospective randomized controlled trials reached a similar conclusion.

Because bedside PDT is logistically simpler and has fewer or equally few complications compared with surgical tracheostomy and is less expensive than surgical tracheostomy in the operating room, the American College of Chest Physicians as well as other national societies recommend PDT as the procedure of choice for performing elective tracheotomy in critically ill adult patients (level 1B). ${ }^{14}$ Thoracic transplantation has been established as an important therapeutic strategy for various end-stage lung or heart diseases. Despite recent refinements of surgical techniques and medical therapy, the mortality is still high, and primary graft failure is considered to be one major cause, especially in the early phase of lung transplantation. Therefore, prolonged mechanical ventilation has to be performed in up to $30 \%$ of all patients after lung transplantation, in the early postoperative time as well as during the long-term course. ${ }^{4}$ Causes of respiratory failure are multifarious and include acute lung injury due to ischemiareperfusion injury, acute graft failure, acute rejection episodes, and pulmonary infections. However, there are only 3 reports in the literature evaluating the safety of PDT in subjects after solid organ transplantation.

Auzinger et al ${ }^{15}$ reported on 16 subjects after lung transplantation who received tracheostomy surgically under general anesthesia in the operating room or percutaneously at the bedside to identify clinical predictors for receiving a tracheostomy after transplant. Variables independently associated with tracheostomy were incidence of pneumonia and need for re-intubation as well as cardiopulmonary bypass time and severity of reperfusion injury. However, no difference existed between the tracheostomy and control groups in terms of short- and long-term survival.

Another study investigated the incidence of complications after PDT in a homogenous population of 51 transplant subjects (17 lung transplants, 32 liver transplants, 2 kidney transplants). ${ }^{6}$ The median age was $55 \mathrm{y}$ (range 27-73 y), median time from intubation to PDT was $10 \mathrm{~d}$, and median time from transplant to PDT was $22 \mathrm{~d}$. Periprocedural complications were observed in 7 subjects, and postprocedural complications were seen in 4 subjects, including bleeding, bradycardia, hypotension, tracheal ring fracture, and cannula malfunction.

The largest series of PDTs in thoracic transplant subjects included 31 subjects after lung transplantation (24.6\% of all lung transplant patients in a 5-y period), including 5 subjects receiving extracorporeal membrane oxygenation therapy undergoing surgical $(19.3 \%)$ or percutaneous tracheostomy $(80.6 \%)$ using different PDT techniques. ${ }^{7}$ There were no major complications during the prothrombin time procedures, but there was 1 postprocedural hemothorax as well as 2 cases of subcutaneous emphysema.

Our study included 28 subjects with a coagulopathy, and none suffered from major bleeding requiring transfusion or surgical intervention. Several studies demonstrated the safety of PDT in different congenital or acquired hematological disorders, including liver cirrhosis and dual antiplatelet therapy, or in subjects with coagulopathy or severe thrombocytopenia after cardiac surgery. ${ }^{15,16}$ Therefore, when performed by experienced personnel, PDT with bronchoscopic guidance has a low bleeding complication rate in patients with coagulopathies. However, we recommend that heparin infusions be temporarily interrupted during the procedure to minimize bleeding complications, and 
coagulation factors should be substituted if necessary and possible.

In patients after median sternotomy, tracheostomy might be associated with an increased risk for mediastinitis with a prolonged hospital stay as well as increased hospital and long term mortality. Previous studies investigating the association between early tracheostomy and deep sternal wound infection after median sternototomy showed divergent results: Whereas Ngaage et al ${ }^{17}$ reported post-sternotomy PDT to be associated with an approximately 3-fold risk for deep sternal wound infections, others could not demonstrate any relationship between tracheostomy and mediastinitis. ${ }^{18}$ In our cohort, none of the 14 subjects undergoing thoracic transplant with median sternotomy developed mediastinitis.

The proportion of subjects who underwent PDT in our study is approximately $30 \%$ and therefore somewhat higher than reported in some studies investigating thoracic transplant subjects. ${ }^{7,15}$ In contrast, other groups published comparable data to ours: Feltriacco ${ }^{5}$ reported that $24.6 \%$ of all lung transplant subjects underwent PDT, whereas 34 of 82 $(41.5 \%)$ lung transplant recipients required postoperative mechanical ventilation of $>21 \mathrm{~d}$ in a recently published series. However, it has to be mentioned that it is difficult to compare different studies investigating PDT after transplant because there is no consensus about the indication and timing of PDT. Accordingly, time on mechanical ventilation before PDT varies significantly between studies and is longer in many series compared with our mean time of $<4 \mathrm{~d}$. This might indicate that we used a more liberal approach to PDT compared with others. Although mean time from intubation to PDT might appear very short with $<4 \mathrm{~d}$, it has to be kept in mind that a significant proportion of subjects underwent reintubation and mean time from surgery to PDT was approximately 13 d. ${ }^{19-21}$

A number of authors have recommended the use of bronchoscopy during percutaneous tracheostomy because it allows direct visualization of the airway during the whole tracheostomy, including placement of the needle entering the trachea, the actual dilation of the tracheostomy site, and the final positioning of the tracheostomy tube. However, there is no clear consensus in the literature about the use of bronchoscopy. There have been few studies comparing percutaneous tracheostomy with and without bronchoscopic guidance. The largest comparative study did not show a difference in complication rates with the use of video bronchoscopy. ${ }^{22}$ In fact, the most significant complication (loss of airway and subsequent cardiac arrest) occurred in the bronchoscopy group. Although there is a lack of evidence in the literature, blind PDT without direct visualization of the trachea might be associated with an increased risk of uncentered tracheal positioning, paratracheal insertion, and posterior tracheal wall laceration or perforation with subsequent potential for increased incidence of complications based on our experience.

In addition to bronchoscopy, ultrasound has become an established diagnostic tool during recent years because it has the potential advantage of identifying vessels in the neck and enabling the clinicians to define the needle path by showing displacement of tissues ahead of the needle. The only randomized, controlled trial assigning a total of 50 subjects to tracheal puncture using either traditional anatomical landmarks or real-time ultrasound guidance demonstrated that ultrasound guidance significantly improved the rate of first-pass puncture and puncture accuracy. ${ }^{23}$ There was a trend toward fewer procedural complications without statistical significance. Other studies support the use of real-time ultrasound guidance during PDT in preventing cranial tracheostomy tube misplacement. ${ }^{24}$ Observational data suggest that preprocedural ultrasound may help to prevent vascular complications and that real-time ultrasound guidance during PDT without bronchoscopy is probably safe, with a high success rate. ${ }^{25}$ Because we established routine pre-PDT ultrasound screening of the ventral neck with special attention to the thyroid gland and subcutaneous vessels in 2013 and did not use it routinely during the study period, we cannot make any statements about the impact of pre-PDT ultrasound on the incidence of bleeding complications in thoracic transplant patients.

\section{Limitations}

This is a retrospective, single-center study with designspecific limitations. With no matched control group, we can conclude that PDT was a safe procedure for lung transplant recipients, but we are unable to answer the question of whether PDT is superior to surgical tracheostomy or prolonged ventilation via a translaryngeal tube. Therefore, our findings should be confirmed in a larger multicenter study.

\section{Conclusions}

In conclusion, we could demonstrate that the use of PDT as standard airway access for prolonged mechanical ventilation in subjects after lung or heart transplant is safe and associated with low complications rates. Bleeding or infectious complications due to immunosuppressive therapy and coagulopathy are rare.

\section{REFERENCES}

1. Ciaglia P, Firsching R, Syniec C. Elective percutaneous dilatational tracheostomy: a new simple bedside procedure: preliminary report. Chest 1985;87(6):715-719. 


\section{Percutaneous Tracheostomy After Thoracic Transplant}

2. Susarla SM, Peacock ZS, Alam HB. Percutaneous dilatational tracheostomy: review of technique and evidence for its use. J Oral Maxillofac Surg 2012;70(1):74-82.

3. Freeman BD, Isabella K, Lin N, Buchman TGA. meta-analysis of prospective trials comparing percutaneous and surgical tracheostomy in critically ill patients. Chest 2000;118(5):1412-1418.

4. Fuehner T, Greer M, Welte T, Gottlieb J. The lung transplant patient in the ICU. Curr Opin Crit Care 2012;18(5):472-478.

5. Feltracco P, Milevoj M, Alberti V, Carollo C, Michieletto E, Rea F, Loy M, Marulli G, Ori C. Early tracheostomy following lung transplantation. Transplant Proc 2011;43(4):1151-1155.

6. Waller EA, Aduen JF, Kramer DJ, Alvarez F, Heckman MG, Crook JE, et al. Safety of percutaneous dilatational tracheostomy with direct bronchoscopic guidance for solid organ allograft recipients. Mayo Clin Proc 2007;82(12):1502-1508.

7. Boasquevisque $\mathrm{CH}$, Yildirim E, Waddel TK, Keshavjee S. Surgical techniques: lung transplant and lung volume reduction. Proc Am Thorac Soc 2009;6(1):66-78.

8. Rumbak MJ, Newton M, Truncale T, Schwartz SW, Adams JW, Hazard PBA. prospective, randomized, study comparing early percutaneous dilational tracheotomy to prolonged translaryngeal intubation (delayed tracheotomy) in critically ill medical patients. Crit Care Med 2004;32(8):1689-1694.

9. Koch T, Hecker B, Hecker A, Brenck F, Preuß M, Schmelzer T, Padberg W, Weigand MA, Klasen J. Early tracheostomy decreases ventilation time but has no impact on mortality of intensive care patients: a randomized study. Langenbecks Arch Surg 2012;397(6): 1001-1008.

10. Young D, Harrison DA, Cuthbertson BH, Rowan K, TracMan Collaborators. Effect of early vs late tracheostomy placement on survival in patients receiving mechanical ventilation: the TracMan randomized trial. JAMA. 2013;309(20):2121-2129.

11. Gomes Silva BN, Andriolo RB, Saconato H, Atallah AN, Valente O. Early versus late tracheostomy for critically ill patients. Cochrane Database Syst Rev 2012;(3):CD007271.

12. Delaney A, Bagshaw SM, Nalos M. Percutaneous dilatational tracheostomy versus surgical tracheostomy in critically ill patients: a systematic review and meta-analysis. Crit Care 2006;10(2):R55.

13. Higgins KM, Punthakee X. Meta-analysis comparison of open versus percutaneous tracheostomy. Laryngoscope 2007;117(3):447-454.

14. De Leyn P, Bedert L, Delcroix M, Depuydt P, Lauwers G, Sokolov $\mathrm{Y}$, et al. Tracheotomy: clinical review and guidelines. Eur J Cardiothorac Surg 2007;32(3):412-421.
15. Auzinger G, O'Callaghan GP, Bernal W, Sizer E, Wendon JA. Percutaneous tracheostomy in patients with severe liver disease and a high incidence of refractory coagulopathy: a prospective trial. Crit Care 2007;11(5):R110.

16. Deppe AC, Kuhn E, Scherner M, Slottosch I, Liakopoulos O, Langebartels $\mathrm{G}$, et al. Coagulation disorders do not increase the risk for bleeding during percutaneous dilatational tracheotomy. Thorac Cardiovasc Surg 2013;61(3):234-239.

17. Ngaage DL, Cale AR, Griffin S, Guvendik L, Cowen ME. Is poststernotomy percutaneous dilatational tracheostomy a predictor for sternal wound infections? Eur J Cardiothorac Surg 2008;33(6):10761079; discussion 1080-1081.

18. Rahmanian PB, Adams DH, Castillo JG, Chikwe J, Filsoufi F. Tracheostomy is not a risk factor for deep sternal wound infection after cardiac surgery. Ann Thorac Surg 2007;84(6):1984-1991.

19. Díaz-Regañón G, Miñambres E, Ruiz A, González-Herrera S, Holanda-Peña M, López-Espadas F. Safety and complications of percutaneous tracheostomy in a cohort of 800 mixed ICU patients. Anaesthesia 2008;63(11):1198-1203.

20. Yavas S, Yagar S, Mavioglu L, Cetin E, Iscan HZ, Ulus AT, Birincioglu CL. Tracheostomy: how and when should it be done in cardiovascular surgery ICU? J Card Surg 2009;24(1):11-18.

21. Vargas M, Servillo G, Arditi E, Brunetti I, Pecunia L, Salami D, et al. Tracheostomy in intensive care unit: a national survey in Italy. Minerva Anestesiol 2013;79(2):156-164.

22. Jackson LS, Davis JW, Kaups KL, Sue LP, Wolfe MM, Bilello JF, Lemaster D. Percutaneous tracheostomy: to bronch or not to bronch: that is the question. J Trauma 2011;71(6):1553-1556.

23. Rudas M, Seppelt I, Herkes R, Hislop R, Rajbhandari D, Weisbrodt L. Traditional landmark versus ultrasound guided tracheal puncture during percutaneous dilatational tracheostomy in adult intensive care patients: a randomised controlled trial. Crit Care 2014;18(5):514.

24. Chacko J, Gagan B, Kumar U, Mundlapudi B. Real-time ultrasound guided percutaneous dilatational tracheostomy with and without bronchoscopic control: an observational study. Minerva Anestesiol 2015; 81(2):166-174.

25. Gobatto AL, Besen BA, Tierno PF, Mendes PV, Cadamuro F, Joelsons D, Melro L, Park M, Malbouisson LM. Comparison between ultrasound- and bronchoscopy-guided percutaneous dilational tracheostomy in critically ill patients: a retrospective cohort study. J Crit Care 2015;30(1):220.e13-e17. doi: 10.1016/ j.jcrc.2014.09.011. 